\title{
Effects of hypothermia on Pavlovian conditioning in the rabbit: II. Heart rate response
}

\author{
LAWRENCE G. STAVA and RALPH B. HUPKA \\ California State University, Long Beach, California 90840
}

\begin{abstract}
The heart rate of 18 New Zealand White rabbits was classically conditioned while the rabbits were in a state of normothermia, moderate hypothermia, and severe hypothermia. The mean conditioned heart rate deceleration during these three temperatures for 45 acquisition trials was $7 \%, 6 \%$, and less than $2 / 10$ of $1 \%$, respectively. The resting heart rate was depressed in the severe hypothermic rabbits, but it did not vary systematically with the conditioned heart rate decreases. Moderate and severe hypothermia were defined as a drop of $5^{\circ} \mathrm{C}$ and $10^{\circ} \mathrm{C}$, respectively, in rectal temperature.
\end{abstract}

The purpose of this experiment was to determine the effects of moderate and severe hypothermia on the Pavlovian conditioned heart rate of the rabbit. The conditioned heart rate response in the rabbit is a deceleration in heart rate (Schneiderman, Smith, Smith, \& Gormezano, 1966) and recent evidence suggests that it is an autonomic response uninfluenced by respiration and skeletal motor movements (Metcalf \& Schneiderman, 1973; Powell, Goldberg, Dauth, Schneiderman, \& Schneiderman, 1972; Schneiderman, VanDercar, Yehle, Manning, Golden, \& Schneiderman, 1969; Yehle, Dauth, \& Schneiderman, 1967).

Resting heart rate was observed in this experiment. On the basis of the reduction in neural activity hypothesis, we expected a depressed resting heart rate in the severe hypothermic rabbits in comparison to the other rabbits. However, the resting heart rate was not expected to vary systematically with the conditioned heart rate decreases if the finding of Shapson and Hupka (1976) was based on a learning factor.

A moderate hypothermic group, defined as a decrease of $5^{\circ} \mathrm{C}$ in rectal temperature from a normal tempe:ature of $37^{\circ} \mathrm{C}$, was included in this study. Previous neurological research indicated that moderate hypothermia induces hyperresponsivity (Suda, Koizumi, \& Brooks, 1957; Weinstein, Kendig, Goldring, \& O'Leary, 1961). This hyperresponsivity is characterized by a loss of specificity of reaction. That is to say, the effect of an entering stimulus spread to more than the usual number of interneurons and motorneurons (Koizumi, Brooks, \& Ushiyama, 1956). Since this process does not appear crucial in a simple classical conditioning task involving the repeated reflexive elicitation of an autonomic response, we

This experiment was conducted by the first author in partial fulfillment of the requirements for the M. A. degree. Lawrence $G$. Stava is now in the PhD program at the University of Kentucky, Lexington, Kentucky. Address reprint requests to Ralph B. Hupka, Department of Psychology, California State University, Long Beach, California 90840. expected similar performance in the moderate hypothermic and normothermic animals.

\section{Method}

Subjects. The subjects were 18 male New Zealand White rabbits 40-50 days old at the beginning of the experiment.

Apparatus. There were only minor changes from Shapson and Hupka (1976). During acquisition training, the rabbits were suspended in a cloth sling to prevent overt body movement which might disrupt the electrocardiographic record.

The heart rate was recorded through stainless steel safety pins attached to the right and left shoulders and to the base of the spine. The signals from these electrodes were amplified through an ac coupler in a Beckman dynograph. The paper speed for recording the electrocardiogram was set at $50 \mathrm{~mm} / \mathrm{sec}$.

The CS was a $1,000 \cdot \mathrm{Hz}$ tone of $2.25 \mathrm{sec}$ duration $(70 \mathrm{~dB}, \mathrm{SPL})$. The US was a $2-\mathrm{mA}$ ac shock, $50 \mathrm{msec}$ in duration, applied through two stainless steel suturing clips attached, as in Shapson and Hupka (1976), to the orbital region of the right eye. The mean intertrial interval was $3 \mathrm{~min}$.

Measurements. Heart rate conditioning was calculated as the percentage of decrease in heart rate during the CS on five test trials in comparison to the resting heart rate preceding the onset of the CS. Heart rate was divided into blocks of five beats. The average of the two blocks immediately preceding the CS was compared to the average of the four blocks occurring after CS onset in all but four cases. During three trials in the severe hypothermic rabbits and one test trial in a normothermic rabbit, the electrocardiogram was distorted to the extent that not all six blocks could be measured. In these cases, no less than two blocks after CS onset and one block preceding CS onset were measured.

Resting heart rate was calculated as the average of the two blocks preced ing CS onset. Rectal temperature was measured after each test trial.

Procedure. Eighteen rabbits were randomly assigned to a moderate and a severe hypothermic group and a normothermic group.

Preparation of the animals was exactly as in Shapson and Hupka (1976). the heart rate electrodes were attached while under anesthesia on the day preceding acquisition training. Hypothermia was induced prior to acquisition training and maintained during acquisition training. A similar procedure was used for the seven rabbits which received extinction training while in a state of hypothermia. Moderate hypothermia was defined as a loss of $5^{\circ} \mathrm{C}$ in rectal temperature to $32^{\circ} \mathrm{C}$ from a normal of $37^{\circ} \mathrm{C}$. The normothermic rabbits were isolated in an inoperative freezer compartment for the same duration as the severe hypothermic rabbits required to drop the rectal temperature to $27^{\circ} \mathrm{C}$. 
Acquisition training consisted of 45 acquisition trials preceded by nine adaptation trials. Five test trials to measure conditioned heart rate decreases occurred at Trial $5,15,25,35$, and 45 . A test trial was defined as an acquisition trial without presentation of the US.

During extinction, the six animals in each of the three acquisition groups were divided into three subgroups so that two animals were extinguished under each of three conditions: normothermia, moderate and severe hypothermia. Because of procedural difficulties and comparability problems among the groups generated by attempting to return the rectal temperature of an animal trained in a condition of severe hypothermia to a normothermic condition, the extinction sessions were run on the day following acquisition training.

Electrocardiographic records were obtained for every trial until a maximum of 40 trials. The extinction session was terminated if an animal produced no CRs on four consecutive trials. The extinction data were calculated in terms of the mean frequency of CRs to extinction. A CR was defined as a heart rate decrease of $2 \%$ or more during the CS.

\section{Results}

Figure 1 depicts the mean percentage of conditioned heart rate decreases during five test trials of rabbits conditioned while in a state of moderate hypothermia, severe hypothermia, and normothermia. The rapid conditioning of the normothermic and moderate hypothermic rabbits contrasts sharply with the performance of the severe hypothermic animals. The difference among the groups in mean conditioned heart rate decreases yielded a statistically significant main effect for temperature groups $[\mathrm{F}(2,15)=11.24, \mathrm{p}<.01]$ and Groups by Trials interaction $[F(8.60)=4.27, p<.05]$. The mean conditioned heart rate decreases, averaged over the five test trials, for the normothermic, moderate, and severe hypothermic groups were 7.73, 5.93, and .16, respectively. Tukey HDS comparisons (Kirk, 1968) of these group means revealed that the normothermic group $[\mathrm{q}(3,15)=5.80, \mathrm{p}<.01]$ and moderate hypothermic group $[q(3,15)=4.89, \quad p<.01]$ each

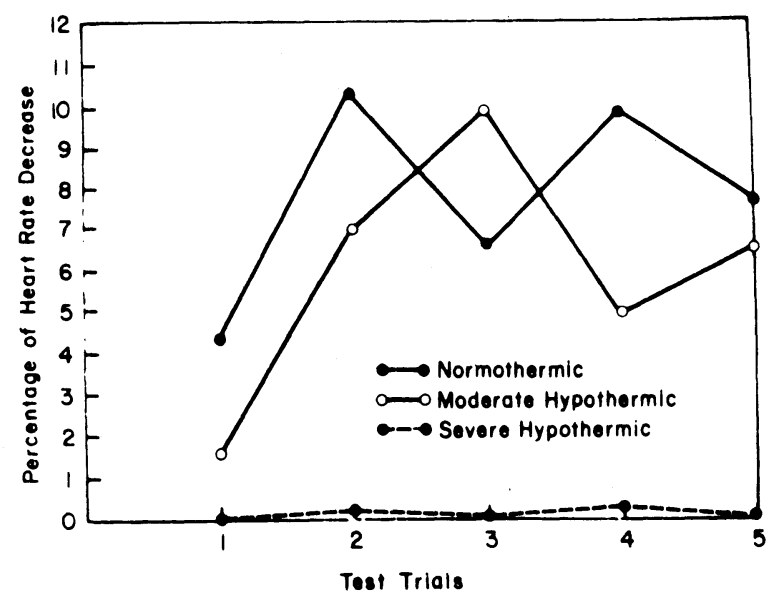

Figure 1. Mean percentage of conditioned heart rate decreases during five test trials of rabbits conditioned while in a state of moderate hypothermia, severe hypothermia, and normothermia.

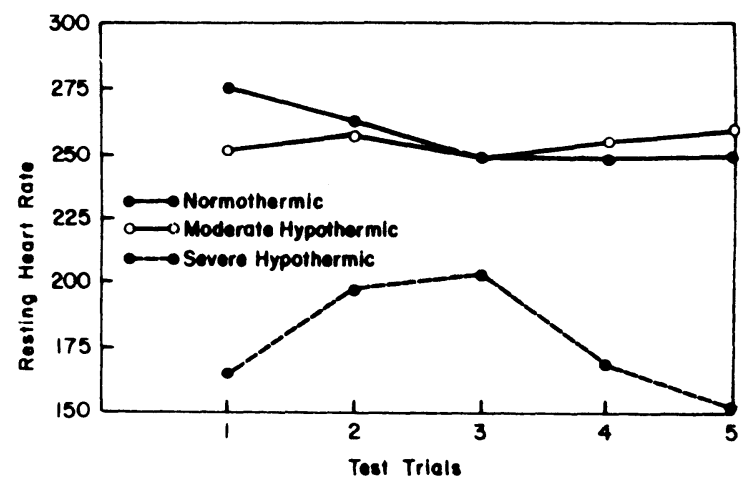

Figure 2. Mean resting heart rate in beats per minute prior to the onset of the CS on five trials for rabbits in a state of moderate hypothermia, severe hypothermia, and normothermia.

differed from the severe hypothermic group, but the normothermic and moderate hypothermic groups did not differ significantly from each other $(\mathrm{q}=1.53)$.

Figure 2 shows the mean resting heart rate in beats per minute for the normothermic, moderate, and severe hypothermic groups prior to CS onset on the five test trials. As expected, the mean resting heart rate was depressed in the severe hypothermic group in comparison to the other two groups $[\mathrm{F}(2,15)=$ $19.72, \mathrm{p}<.01]$. The mean resting heart rate prior to CS onset on the five test trials for the normothermic, moderate, and severe hypothermic groups was 256.97 , 254.16 , and 177.99 , respectively. The normothermic group $[\mathrm{q}(3,15)=7.82, \mathrm{p}<.01]$ and moderate hypothermic group $[q(3,15)=7.54, p<.01]$ each differed significantly from the severe hypothermic group but they did not differ significantly from each other $(\mathrm{q}=.28)$.

Inspection of Figures 1 and 2 clearly indicates that the mean resting heart rate did not systematically vary with the mean percentage of conditioned heart rate decreases. This observation is supported by a correlation coefficient of .34 computed between the heart rate and CR performance for each rabbit for all conditioning trials.

The extinction data were obtained for only 12 of the 18 rabbits because of experimenter error, thus making these data suggestive, rather than definitive,

Table 1

Mean Frequencies of CRs to Extinction for Rabbits Trained and Extinguished While in Normothermia, Moderate and Severe Hypothermia

\begin{tabular}{lccc}
\hline Acquisition & \multicolumn{3}{c}{ Extinction Temperature } \\
Temperature & Normal & Moderate & Severe \\
\hline Normal & $33^{*}$ & $22^{*}$ & $0 \dagger$ \\
Moderate & $18 \dagger$ & $7^{*}$ & $1^{*}$ \\
Severe & $1.5 \dagger$ & $0^{*}$ & $0^{*}$ \\
\hline & $*_{n=1}$ & $t_{n}=2$
\end{tabular}


in nature. Listed in Table 1 are the mean frequencies of CRs to extinction for rabbits conditioned at one of three temperatures and then extinguished at one of the three temperatures. Inspection of Table 1 reveals that extinction is more rapid the more the rectal temperature during extinction deviates from the normal temperature. Rabbits trained in either moderate or severe hypothermia gave more CRs when extinguished while in normothermia than when extinguished while in their respective acquisition training temperatures.

\section{DISCUSSION}

Less than an average of $2 / 10$ of $1 \%$ of conditioned heart rate deceleration occurred in the severe hypothermic rabbits in contrast to the average $6 \% \mathrm{CR}$ deceleration in the moderate hypothermic rabbits and the average $7 \%$ CR heart deceleration in the normothermic rabbits. In agreement with previous findings that severe hypothermia diminishes neural activity (Callaghan, McQueen, Scott, \& Bigelow, 1954; Sarajas \& Putkonen, 1968; Suda et al., 1957), the resting heart rate was depressed in the severe hypothermic rabbits in comparison to the other rabbits. However, it is unlikely that the depressed resting heart rate was directly responsible for the minimal conditioning in the severe hypothermic animals because the resting heart rate did not vary systematically with the conditioned heart rate decreases. Nor is it likely that the poor CR performance of the severe hypothermic animals was due to the operation of the reciprocal of the law of initial values. That is to say. it is not likely that the performance of these animals was due to a physiological limit already having been reached by the slow resting heart rate beyond which further decreases in heart rate were not possible. because on two consecutive test trials one of the hypothermic rabbits had a faster resting heart rate than the normothermic animal being run at the same time. The normothermic, but not the hypothermic rabbit. gave CRs.

Failure to obtain substantial heart rate conditioning in the severe hypothermic rabbits is taken by us as evidence that the equally poor CR performance in conditioning the nictitating membrane represents a learning factor rather than an inability to execute a response. Our findings with a Pavlovian paradigm and the findings of others with instrumental paradigms (e.g., Essman \& Sudak, 1963) provide behavioral support for what also is found at the level of physiological and neurological explorations of the effects of hypothermia (e.g.. Sarajas \& Putkonen, 1968; Suda et al., 1957). Whether the learning deficits obtained during hypothermia are ultimately demonstrated to be in response to depressed neural activity. disruptions of central nervous system functions, delays in fixation of the memory trace, or brain seizure activity are exciting issues awaiting further research.

\section{REFERENCES}

Callaghan, J., McQueen, D., Scott, J., \& Bigelow, W. G. Cerebral effects of experimental hypothermia. Archives of Surgery, 1954, 68, 208.

Essman, W. B., \& Sudak, F. Effect of hypothermia on the establishment of a conditioned avoidance response in mice. Journal of Comparative and Physiological Psychology, 1963, 56, 366-369.

KIRK, R. E. Experimental design: Procedures for the behavioral sciences. Belmont, California: Wadsworth, 1968.

Koizumi, K., Brooks, C., \& Ushiyama, J. Hypothermia and reaction patterns of the nervous system. Annals of the New York Academy of Sciences, 1956, 80, 449-456.

Metcalf, F. U., JR., \& Schneiderman, N. Effects of 6-hydroxydopamine on conditioned and unconditioned cardiovascular responses in rabbits. Proceedings of the 81st Annual Convention of the American Psychological Association, 1973, 8, 1055-1056.

Powell, D. A., Goldberg, S. R., Dauth, G. W., Schneiderman, E., \& Schneiderman, N. Adrenergic and cholinergic blockade of cardiovascular responses to subcortical electrical stimulation in unanesthetized rabbits. Physiology and Behavior, 1972, 8, 927-936.

Sarajas, H. S. S., \& Putkonen, P. T. S. Central nervous and visceral reaction patterns in rabbits warming up from deep hypothermia. Annales Academica Scientiarium Fennicae, 1968, 144, 3-21.

Schneiderman, N., Smith, M. C., Smith, A. C., \& Gormezano, I. Heart rate classical conditioning in rabbits. Psychonomic Science, 1966, 6, 241-242.

Schneiderman, N., VanDercar, D. H., Yehle, A. L. Manning, A. A., Golden, T., \& Schneiderman, E. Vasal compensatory adjustment: Relationship to heart rate classical conditioning in rabbits. Journal of Comparative and Physiological Psychology, 1969, 68, 175-183.

Shapson, D. B., \&. Hupka, R. B. Effects of hypothermia on Pavlovian conditioning in the rabbit: I. Nictitating membrane response. Bulletin of the Psychonomic Society, 1976, 7, 243-245.

SudA, I., KoIzumi, K., \& Brooks, C. Analysis of effects of hypothermia on central nervous system responses. American Journal of Physiology, 1957, 189, 373-380.

Weinstein, J., Kendig, J., Goldring, S., \& O'Leary, J. Hypothermia and electrical activity of cerebral cortex. Archives of Neurology, 1961, 4, 441-448.

Yehle, A. L., Dauth, G., \& Schneiderman, N. Correlates of heart-rate classical conditioning in curarized rabbits. Journal of Comparative and Physiological Psychology, 1967, 64, 98-104.

(Received for publication October 20, 1975.) 